\title{
Erratum
}

\section{Erratum: Palenciano et al., "Representational Organization of Novel Task Sets during Proactive Encoding”}

In the article "Representational Organization of Novel Task Sets during Proactive Encoding" by Ana F. Palenciano, Carlos GonzálezGarcía, Juan E. Arco, Luiz Pessoa, and Maria Ruz, which appeared on pages 8386-8397 of the October 16, 2019 issue, the legend within Figure 3 contained a misprint. The labels for "Across-Dim." and "Within-Dim." were switched. The same mistake applies for "Sequential" and "Single" labels. These errors affected only the figure and not the results provided in the Behavioral results section of the article. Consequently, it does not affect the conclusions of the study. The authors regret this error. The figure has been corrected in the online version and a corrected version of Figure 3 is displayed below.

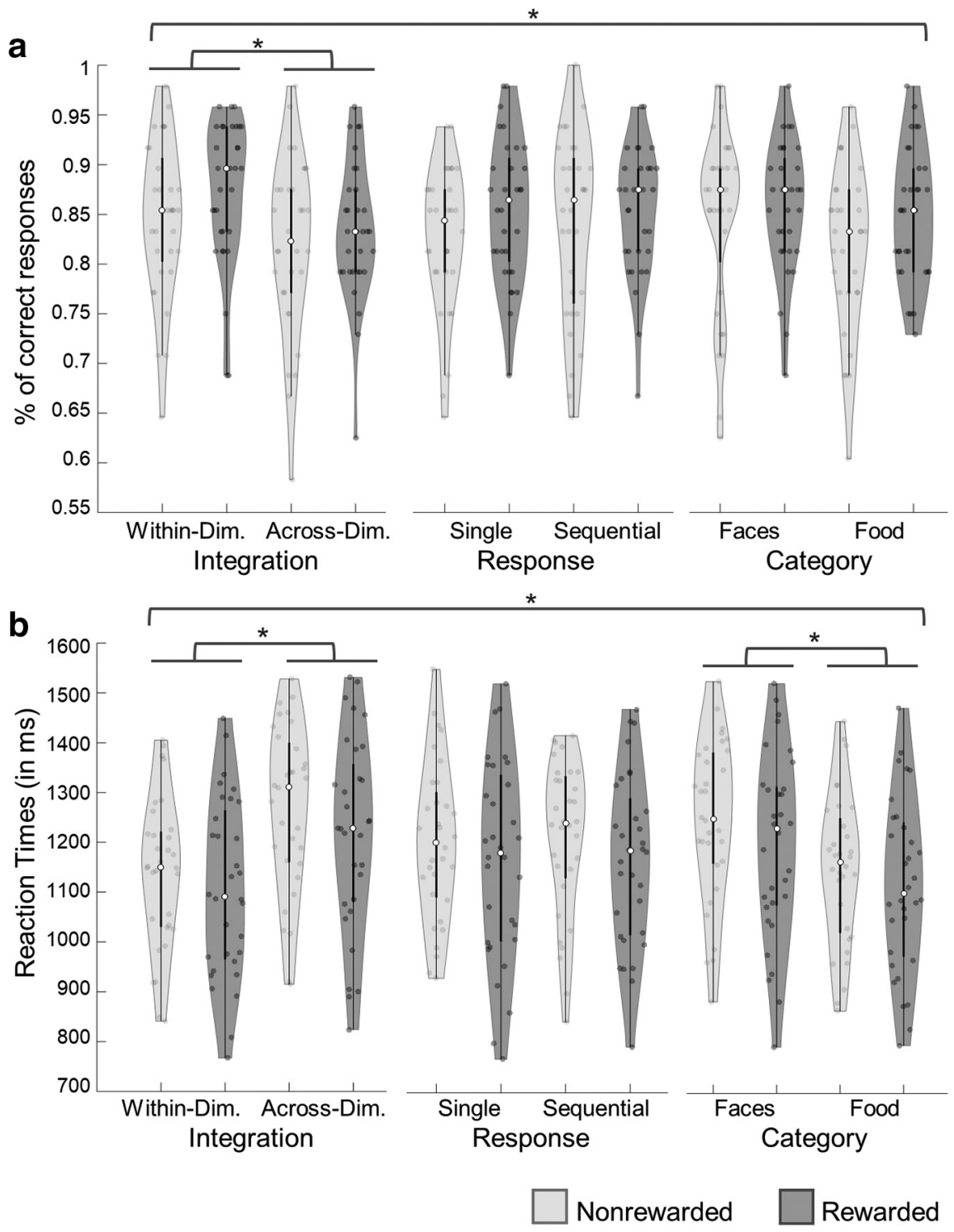

Figure 3. 